\title{
Etude hydrobiologique de la vallée d'Ossau (Pyrénées- Atlantiques, France) III. Simuliidae (Diptera, Nematocera) : leur originalité biogéo- graphique et écologique
}

\author{
G. Vinçon 1 \\ M. Clergue-Gazeau1
}

Mots clés : Simuliidae, biogeographie, écologie, Pyrénées.

Les auteurs présentent une liste de 25 espèces de Simulies récoltées dans les réseaux hydrographiques du Gave d'Ossau et du Haut Rio Gallego (entre 450 et $2150 \mathrm{~m}$ d'altitude). Quelques précisions biogéographiques et écologiques sont données. Deux analyses factorielles des correspondances précisent les relations entre les principaux facteurs de répartition en montagne et mettent en évidence trois groupements d'espèces, confirmés également par la méthode des nuées dynamiques.

Une classification hiérarchique des espèces complète ces analyses.

\begin{abstract}
A hydrobiological study of the Ossau valley (At lantic Pyrenees, France). III. The Simuliddae (Diptera, Nematocera) : their biogeographical and ecological originality.

Keywords : Simuliidae, biogeography, ecology, Pyrenees.

25 species of Simuliidae were collected in the hydrographical system of the Gave d'Ossau and the Haut Rio Gallego (between altitudes of 450 and $2150 \mathrm{~m}$ ). Several biogeographical and ecological data are given. Two factor analyses revealed relationships between the principal factors affecting distribution in the mountains, and showed that there were three groups of species, a conclusion also confirmed by cluster analysis.
\end{abstract}

\section{Introduction}

Les Simulies des Pyrénées occidentales sont peu connues ; les deux seules notes ayant trait à l'écologie de ces Nématocères concernent des cours d'eau de basse altitude : le Lissuraga $(50-400 \mathrm{~m}$ : Neveu 1970) et la Basse Nivelle (au-dessous de $50 \mathrm{~m}$ : Neveu et Lapchin 1979) ; il était donc intéressant de prospecter un réseau de haute montagne.

Des recherches ont été entreprises depuis quatre ans sur l'ensemble de la faune macroinvertébrée du

1. Laboratoire d'Hydrobiologie, UA CNRS 695, Úniversité Paut Sabatier. 118, Route de Narbonne, 31062 Toulouse Cédex, France. réseau hydrographique de deux vallées situées dans la même région: la vallée d'Ossau (versant Nord: $450-2150 \mathrm{~m}$ ) et la haute vallée du Rio Gallego (versant Sud : $1740-1750 \mathrm{~m}$ ) dans le but d'élaborer une méthodologie de surveillance des cours d'eau de montagne (Vinçon 1987 a). La présente note précise la répartition géographique des Simuliidae de cette région et analyse le peuplement à partir de données semi-quantitatives.

\section{Les stations étudiées}

L'échantillonnage a été effectué dans 29 stations - 27 sur le réseau du Gave d'Ossau et 2 sur le Rio Gallego, (fig. 1); elles représentent des biotopes 


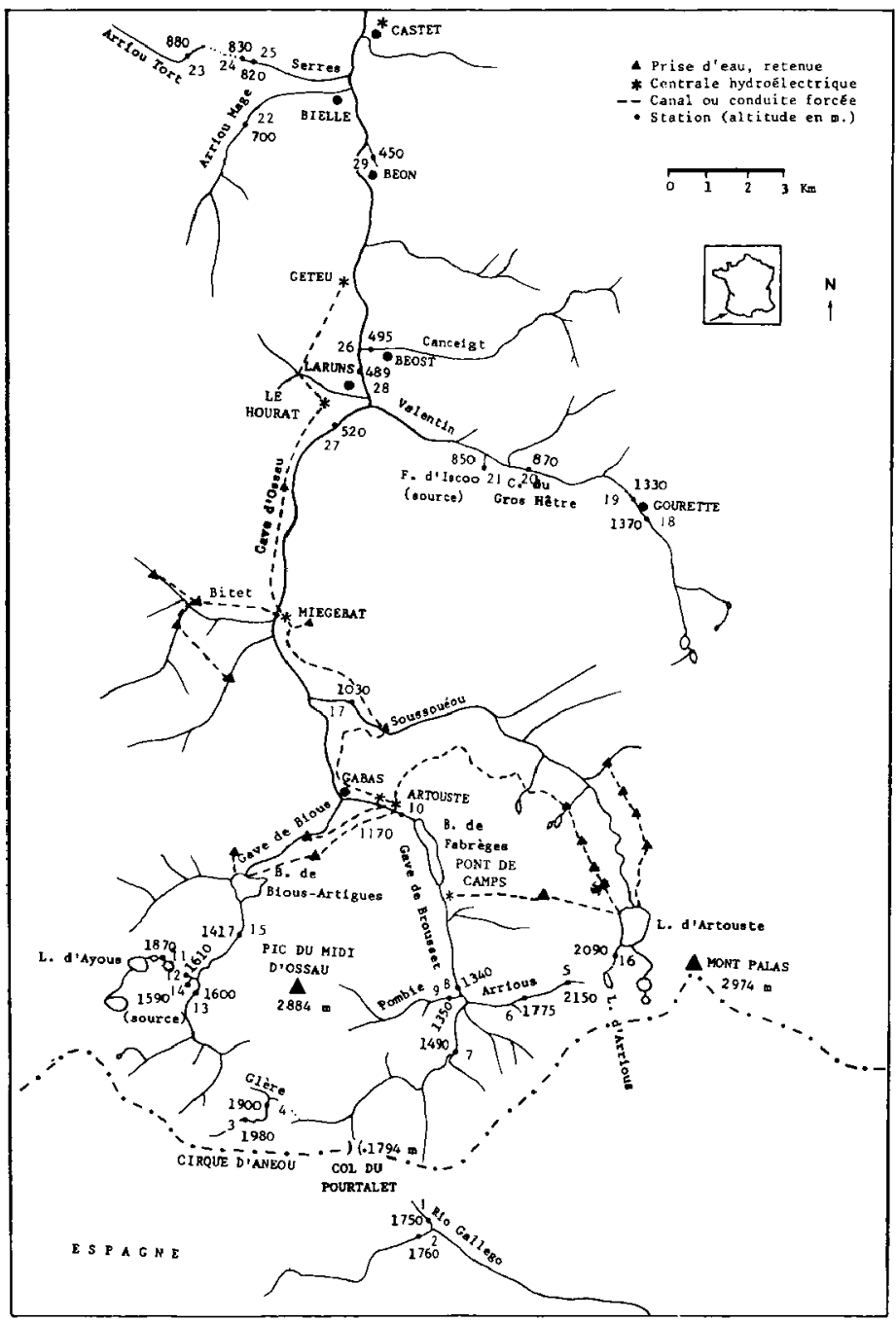

Fig. 1. Réseau hydrographique du gave d'Ossau.

Stations et principaux aménagements hydro-électriques connus. 
variés. Un code est attribué à chacune d'elles et comprend les initiales de la station, son altitude et son numéro.

La liste des stations est la suivante:

Vallée du Rio Gallego

1. Rio Gallego

2. affluent du Rio Gallego

R.G. 1750

A.R.G. 1760

Vallée d'Ossau

3. affluent de la Glère

A.G. 1980

4. affluent de la Glère (méandres)

5. ruisseat d'Arrious (source)

6. ruisseau d'Arrious

7. Gave de Brousset

8. Gave de Brousset (amont barrage Fabrèges)

A.G. 1900

Ar. 2150

Ar. 1775

Br. 1490

Br. 1350

P. 1350

9. ruisseau de Pombie

10. Gave de Brousset (aval barrage)

11. déversoir du lac d'Ayous (amont lac Roumassot)

12. affluent du Gave de Bious

13. Gave de Bious

14. source affluent du Gave de Bious (résurgence karstique)

Br. 1170

D.Ay. 1870

A.Bi. 1610

Bi. 1600

15. Gave de Bious

S.A.Bi. 1590

Bi. 1417

Ar. 2090

S. 1030

17. Soussouéou au pont du Goua

V. 1370 (amont de Gourette)

19. torrent du Valentin (aval de Gourette) V. 1330
20. torrent du Valentin (Cascade du Gros Hêtre)

V. 870

21. source d'Iscoo (résurgence karstique) S.I. 850

22. torrent d'Arriou Mage

A.M. 700

23. ruisseau lent d'Arriou Tort

A.T. 880

24. ruisseau calcaire de Serres (prairie) Se. 830

25. ruisseau calcaire de Serres (hêtraie) Se. 820

26. Gave d'Ossau (amont de Laruns)

O. 520

27. torrent du Canceigt

C. 495

28. Gave d'Ossau (aval de Laruns)

O. 489

29. ruisseau au Nord de Béon "Miédougué " (résurgences karstiques de pied de massif)

R.N.B. 450

Les principaux paramètres de ces stations sont donnés dans le tableau I. Le détail en est précisé dans un article de présentation du milieu (Vinçon $1987 \mathrm{~b}$ ).
Tableau I. Paramètres des stations Les stations étudiées en vallée d'Ossau et dans le Rio Gallego: leurs caractéristiques.

\begin{tabular}{|c|c|c|c|c|c|c|c|c|c|}
\hline $\begin{array}{l}\text { Stations, } \\
\text { dltitude (m), } \\
\text { et } n^{\circ} \text { de code }\end{array}$ & 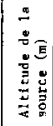 & $\begin{array}{l}\hat{u} \\
\dot{0} \\
\dot{y} \\
\dot{E} \\
\dot{z}\end{array}$ & 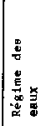 & 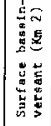 & 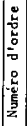 & 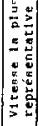 & 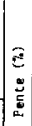 & 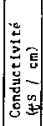 & 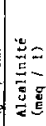 \\
\hline $.2150(5)$ & 30 & & & 0,3 & 1 & $M$ & 30 & 76 & 0,4 \\
\hline Ar . 2090 & 250 & 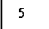 & & 0,2 & 1 & L & 3 & 76 & 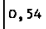 \\
\hline c. 1980 (3) & 80 & 15 & $\mathrm{NT}$ & 1,1 & 1 & $\mathrm{TR}$ & 15 & 52 & 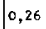 \\
\hline .G. $1900(4)$ & 80 & 16 & & t. & 1 & $M$ & 5 & 95 & 0,6 \\
\hline ay. & 70 & 16 & & 1,8 & 2 & Tr & 25 & 54 & 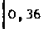 \\
\hline$=1775(6)$ & 2230 & 9 & & 2 & 2 & & 10 & 84 & $10,6\}$ \\
\hline R.G. & 1900 & 15 & $\mathrm{NT}$ & 0,8 & 1 & & 10 & 75 & 0,5 \\
\hline .G. 17 & 1800 & 15 & NT & 0,3 & 1 & H & 5 & 172 & 1,18 \\
\hline 1. 1 & 1650 & 16 & $N T$ & & 1 & TR & 30 & 55 & 0,3 \\
\hline 1. $1600(23)$ & 2200 & 10 & NI & 12 & 3 & TR & 20 & 124 & 0,98 \\
\hline$A-B S$. & 90 & 6 & & 0,2 & o & M & 20 & 184 & 1,4 \\
\hline 3r. 14900 & 2080 & 14 & NT & 17 & 3 & TR & 10 & 163 & 1,21 \\
\hline 11. 1417 & $\infty$ & 1 & & 1 & 3 & $T R$ & 15 & 107 & 0,86 \\
\hline . $1370<1$ & 2280 & 1 & T & 7 & 3 & $\mathrm{TR}$ & 10 & 82 & 0,88 \\
\hline $.1350(9)$ & 00 & & & 6 & $2^{2}$ & TR & 20 & 73 & 0.50 \\
\hline r. $1340(8)$ & 230 & 13 & $T$ & 22 & 3 & $R$ & 5 & 139 & $1, \infty$ \\
\hline - 13300 & 280 & 1 & & 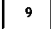 & 3 & $T R$ & 10 & 168 & 1,3 \\
\hline$x=1$ & 2230 & 13, & $\mathrm{NT}$ & 61 & 3 & R & 8 & 156 & 1,20 \\
\hline $1030(1$ & 320 & & & 52 & 3 & TR & 15 & 90 & $\{0,60$ \\
\hline T. 880 & 950 & 2 & PN & 9 & 1 & , & 1 & 283 & 2,48 \\
\hline $870(20$ & 2280 & 1 & & 16 & 3 & . & 5 & 195 & 1,47 \\
\hline . I. 850 & 850 & 8 & & 0,3 & 0 & $R$ & 30 & 218 & 1,86 \\
\hline .830 & 950 & 1 & . & 9 & 1 & ${ }^{2}$ & 2 & 402 & 3,68 \\
\hline e. $820(25)$ & 950 & 17 & $\mathrm{PN}$ & 9,5 & 2 & m & 10 & 351 & 3,36 \\
\hline$. M .700<22$ & 550 & 1 & $\cdots$ & 15 & 2 & IR & 15 & 219 & 1,86 \\
\hline $.520(27)$ & & & 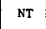 & 220 & 4 & $L_{0}$ & 3 & 201 & 1,63 \\
\hline $495(26)$ & & 1 & 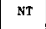 & 22 & 3 & 8 & 8 & 287 & 1,94 \\
\hline $489(28)$ & & 15 & NT & 280 & 4 & " & 1 & 190 & 1,63 \\
\hline .N.B. 450 (29) & $\infty$ & , & $s$ & 0,2 & | & I & 2 & 228 & 1,96 \\
\hline
\end{tabular}

\section{La faune}

\subsection{Méthodes et périodes de récoltes}

La grande diversité des milieux interdisait toute étude quantitative stricte; aussi, afin d'obtenir des données qualitatives comparables, chaque station a-1-elle été prospectée pendant des laps de temps identiques. Une station correspond à un tronçon d'une longueur voisine de $50 \mathrm{~m}$; elle regroupe un ensemble d'habitats : pierres en plein courant, zones calmes, végétation immergée... 
Les déterminations ont été réalisées sur du matériel récolté par dérives (larves et exuvies nymphales) et par prélèvements benthiques (filet Surber dans le courant). Les nymphes sont détachées des rochers à la pince ou prélevées sur la végétation.

Dans certains cas, les déterminations ont pu être confirmées ou complétées par la capture d'adultes au filet entomologique.

Afin d'obtenir une liste faunistique représentative du peuplement simulidien en montagne, il est indispensable de prospecter durant les quatre périodes suivantes : avril, juin, deuxième quinzaine de juillet, début octobre. En fait, sept campagnes de récoltes ont été réalisées pendant l'année 1983-1984 : dérives (D), prélèvements benthiques (B) et chasses d'adultes (A).

du $18 / 11 / 83$ au $23 / 11$ : D.B.A.

du $15 / 03 / 84$ au $17 / 03$ : D.B.A.

du 30/04 au 06/05: A.

du 11/06 au 15/06: A.

du $26 / 07$ au $2 / 08$ : D.B.A.

du $27 / 09$ au $2 / 10$ : D.B.A.

du $7 / 11$ au 12/11: A.

\subsection{Liste faunistique}

25 taxons et 2 formes ont été recensés dans ces cours d'eau ; ils sont énumérés dans le tableau II, suivant la classification de Crosskey (1981).

L'abondance relative de chaque espèce est notée en utilisant 5 classes correspondant aux récoltes cumulées :

classe 1 : un seul individu capturé. Cette classe indique une présence très rare ou, à la limite, incertaine. classe $2: 2$ à 4 individus (espèce rare)

classe $3: 5$ à 30 individus (espèce assez abondante) classe $4: 31$ à 100 individus (espèce abondante) classe 5: supérieur à 100 individus (espèce très abondante).

\subsection{Remarques sur quelques espèces}

Les taxons recensés sont en majorité bien connus et largement répandus dans la zone paléarctique (Zwick 1978) ; néanmoins, nous présentons ici quelques particularités concernant la biogéographie, l'écologie ou la morphologie de certaines espèces.

\section{P. (P.) tomosvaryi}

Rencontrée dans sept de nos stations dont trois sont des sources (résurgences karstiques), elle présente par conséquent une affinité pour le crênal, ce qui correspond aux observations de $\mathrm{H}$. Zwick (1974, p. 47) dans le réseau de la Fulda.

Cette espèce colonise aussi des ruisseaux de très haute altitude: le déversoir du lac d'Arrious à $2090 \mathrm{~m}$ en vallée d'Ossau (st. 16) et plusieurs affluents du Sègre en Andorre (1 240-2 280 m) (González 1980).

Dans le bassin du Lot, au sud du Massif Central, elle est surtout abondante au-dessus de $1000 \mathrm{~m}$ et serait de ce fait sténotherme d'eau froide (Gagneur 1976).

Toutefois, dans les Pyrénées-Orientales, elle a été capturée tout le long de la rivière Massane jusque dans les zones riches en végétation aquatique de basse altitude (Doby \& Deblock 1955 et ClergueGazeau 1987), ce qui montre qu'elle peut supporter des températures estivales élevées $\left(20^{\circ} \mathrm{C}\right.$ à $50 \mathrm{~m}$ d'altitude). Des prélèvements dans d'autres vallées nous semblent encore nécessaires pour préciser son écologie.

\section{S. (N.) latigonitum}

Signalée de 3 stations en vallée d'Ossau, l'espèce est particulièrement bien représentée dans le ruisseau de Serres, biotope caractérisé par son courant lent, sa richesse en matières organiques, ses fortes teneurs en calcium et ses températures estivales éle. vées. Mise à part la teneur en calcium, il semble que ce type de milieu soit son biotope préférentiel puisqu'elle a été capturée dans les mêmes conditions à Thuès-les-Bains : $750 \mathrm{~m}$ (Jarry 1975), seule station connue jusqu'à maintenant dans les Pyrénées, ainsi que dans le Nord-Ouest de l'Espagne (altitude inférieure à $600 \mathrm{~m}$ : Beaucournu-Saguez 1975).

\section{S. (Od.) gr. ornatum}

Les larves récoltées dans le Rio Gallego (1 $750 \mathrm{~m})$ présentent des particularités dans la forme ramifiée des branchies anales. Un plus ample matériel serait nécessaire (notamment des mâles) pour préciser la position taxonomique de cette forme dans le sousgenre Odagmia. Beaucournu-Saguez (1975) signale également des branchies anales ramifiées sur des larves de ce groupe dans le Nord-Ouest de l'Espagne.

\section{S. (S.) gr. monticola}

Contrairement à la vallée d'Aure où prédomine $S$. (S.) argyreatum à plus de $90 \%$, les deux espèces $S$. (S.) argyreatum et $S$. (S.) monticola sont ici aussi 
Tableau II. Répartition des Simuliidae en vallée d’Ossau.

Classes d'abondance indiquées sur le tableau.

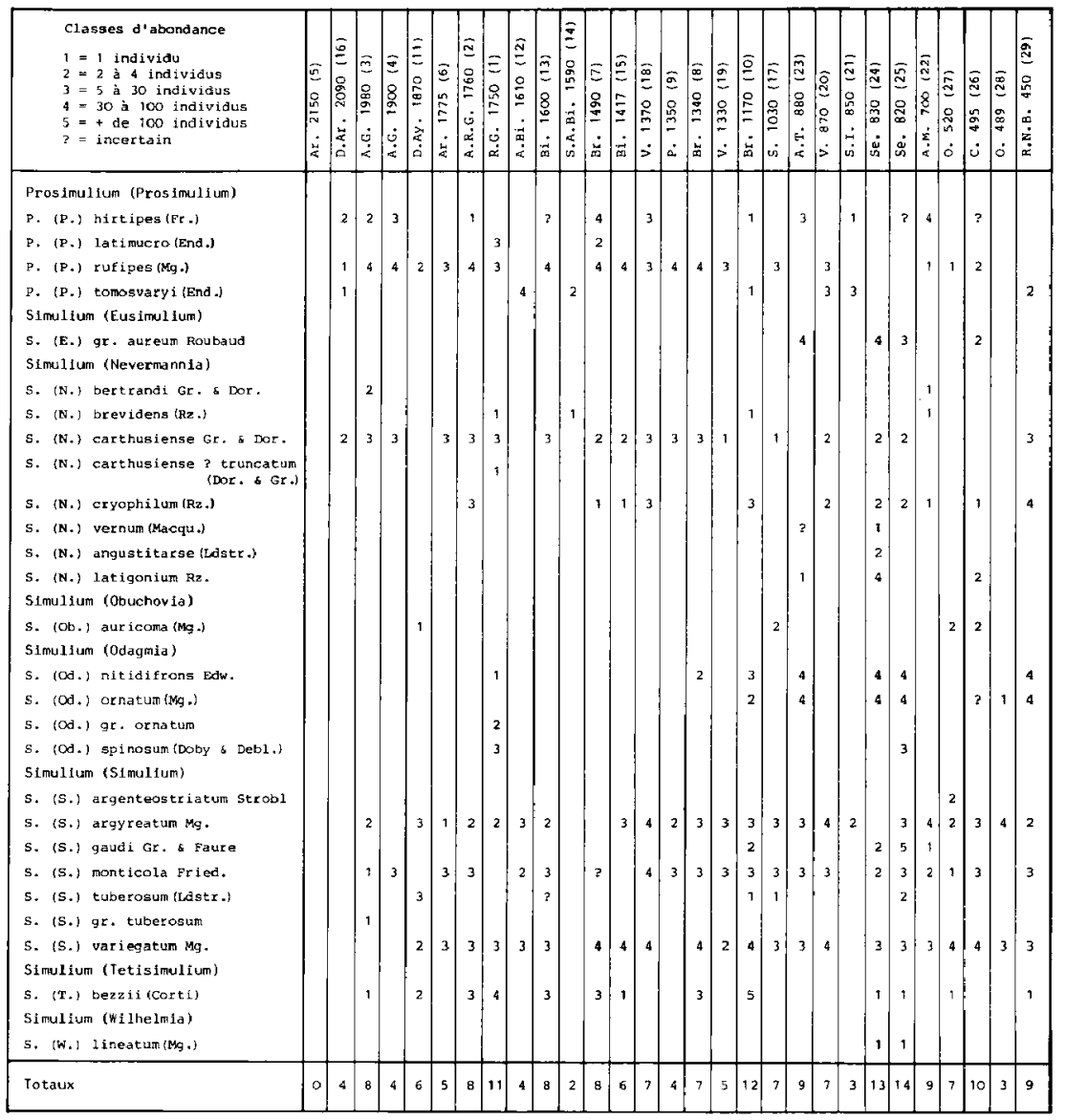


fréquentes et abondantes l'une que l'autre, el occupeni apparemment les mêmes biotopes.

\section{gr. argenteostriatum}

D'après nos résultats, l'espèce-type $S$. (S.) argenteostriatum est très rare dans cette vallée (2 nymphes à $520 \mathrm{~m}$ ). En revanche, elle est fréquente dans les Pyrénées centrales entre 400 et $2000 \mathrm{~m}$. tout en restant faiblement abondante, d'après Lavandier (1976 et 1979) et Gazagnes (1983).

L'autre espèce du même groupe : $S$. ( $S$, hispaniola. a été capturée récemment en basse altitude dans une vallée située encore plus à l'Ouest : celle du Saison. Elle y est très abondante, omniprésente sur une trentaine de kilometres en amont de la ville de TardetsSorholus (alt. $220 \mathrm{~m}$ ), ce parcours représentant une faible dénivellation ( $150 \mathrm{~m}$ ). Ces données confirment les observations de Doby, David et Rault (1964) qui obtiennent une moyenne de $1000 \mathrm{~m}$ d'altitude pour S. (S.) argenteostriatum, plutôt alticole, alors qu'elle in de $400 \mathrm{~m}$ pour $S$. (S.) hispaniola, espece de piémont.

\section{S. (S.) gaudi}

Nouveau pour les Pyrénées, ce taxon a été rencontré dans 4 stations de moyenne altitude $(820-1170 \mathrm{~m})$; il est particulièrement abondant dans le ruisseau calcaire de Serres $(820-830 \mathrm{~m})$ et caractérise ce biotope au même titre que d'autres Diptè. res calcicoles : Lithotanytarsus emarginatus (Chironomidae), Pericoma barbarica et $P$. calcilega (Psychodidae)...

Récemment, il a été retrouvé dans un affluent de la basse Neste d'Aure : le ruisseau d'Esponne, qui coule sur des affleurements calcaires (ClergueGazeau, Holmière, Meurgues et Angelier 1987).

\section{S. (W.) lineatum}

Cet élément vit habituellement dans les fleuves de plaine à végétation plus ou moins abondante. Sa présence en moyenne al titude $(820 \mathrm{~m})$ est toutefois possible dans des ruisseaux lents, riches en matières organiques et soumis à des températures estivales élevées, comme le ruisseau de Serres. Dans le SudEst de la France, Dorier (1962-63) l'avait déjà signalée de milieux semblables, au-dessus de $500 \mathrm{~m}$.

\subsection{Les Simulies dans la chaîne pyrénéenne}

Ayant regroupé les données faunistiques provenant de plusieurs vallées pyrénéennes: Ossau et haut Gallego à l'Ouest, Neste d'Aure dans la partie centrale (Lavandier 1976 et 1979, Clergue-Gazeau et Gazagnes 1986 et Clergue-Gazeau et al. 1987) et haute vallée du Sègre à l'Est (González-Peña 1980 et 1983), nous pouvons en comparer les peuplements (Tableau III). Une analyse de ces données, auxquelles peuvent être ajoutées celles de la Massane (Clergue-Gazeau 1987), dans la partie orientale, permet de faire plusieurs observations.

\section{EN HAUTE MONTAGNE}

Certaines espèces telles que $S$. (S.) variegatum, $P$. (P.) hiripes, S. (T.) bezzii dépassent $1700 \mathrm{~m}$ dans les vallées du Sègre, d'Ossau ou du Gallego alors qu'elles sont très rares ou absentes au-dessus de $1200 \mathrm{~m}$ en vallée d'Aure. La montée de ces espèces en haute altitude est sans doute une conséquence des influences maritimes qui temperent le climat dans la vallée d'Ossau (versant Nord) mais aussi une conséquence de l'exposition Sud des bassins. versants du Sègre et du Gallego. Ces observations sont confirmées en outre par:

a) les travaux de Jarry (1973) qui note la présence de $S$. (S.) variegatum et $S$. (T.) bezzii au-dessus de $2000 \mathrm{~m}$ dans la vallée de la Têt (PyrénéesOrientales) ;

b) les travaux de Clergue-Gazeau et Gazagnes (1986) et Clergue-Gazeau et al (1987) qui indiquent pour ces mêmes especes une colonisation très importante des zones de basse et moyenne montagne avec présence très sporadique au-dessus de $1200 \mathrm{~m}$, dans la partie centrale des Pyrénées.

Certaines espèces eurythermes, fréquentant les zones de basse et moyenne montagne du centre de la chaîne, at teignent par consequent dans les parties Ouest et Est des altitudes plus élevées, ce qui est observé également pour d'autres familles de macroinvertébrés (Plécoptères notamment : Vinçon 1987a p. 151).

\section{EN ZONE DE PIEMONT}

La basse vallée de la Neste possède une richesse spécifique plus importante (26 éléments) que la basse vallée d'Ossau (19). Ce fait est dú probablement à des conditions thermiques particulières : dans sa partie aval (Beyrede $650 \mathrm{~m}$ ), une partie des eaux de la Neste est détournée dans un canal d'irrigation, d'où une diminution de la profondeur de la rivière qui s'étale sur une grande largeur. De ce fait, elle subit un réchauffement rapide favorable au 
Tibleau III. Répartition altitudinale des Simuliidae recensées dans plusieurs vallées pyrénéennes. Trait discontinu : vall. lée du Sègre ; traił plein : vallée d'Aure : pointillé : vallée d'Ossau ; étoile noire : haute vallée du Rio Gallego ; deur étoiles noires : détermination incertaine dans la vallée du Sègre.

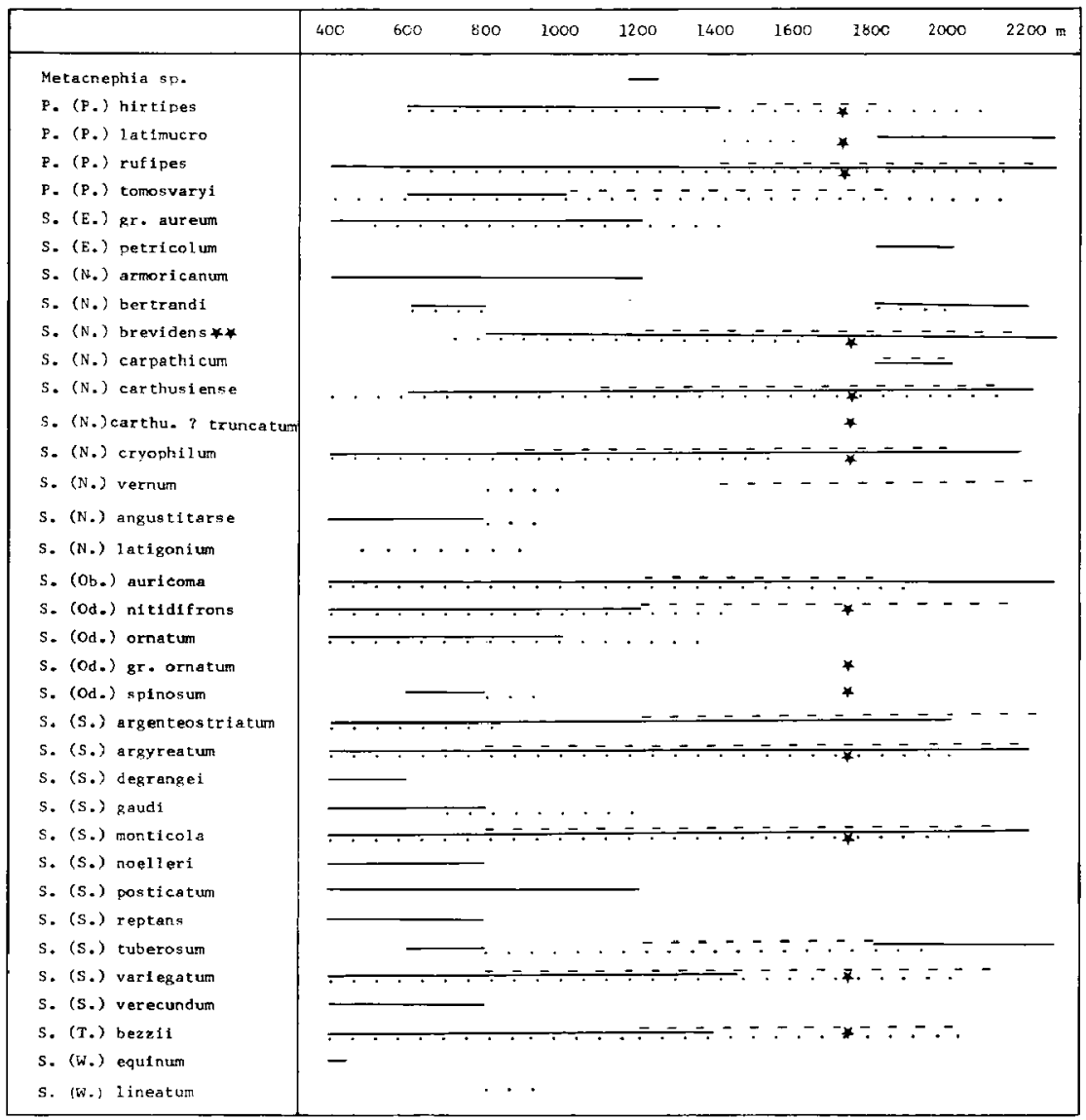


développement d'espèces à tendance thermophile, telles que $S$. (S.) reptans, $S$. (S.) verecundun... La première colonise abondamment toute la basse vallée : son abondance diminue progressivement en remontant vers la moyenne vallée et elle disparaît en amont de la dérivation du canal d'irrigation, au moment où le cours d'eau retrouve son aspect tor. rentiel avec lit plus étroit. La même observation a été faite pour les Hydracariens de la basse vallée d'Aure (Angelier et al. 1985).

\subsection{Influences humaines sur la faune}

a) D'après les observations effectuées en amont et en aval du barrage de Fabrèges (st. 8 et 10 ), les éléments sténothermes d'eau froide tels que $S$. $(N$.) carthusierise et $P$. (P.) rufipes, présents en amont (st. 8 ), sont remplacés vers l'aval par $P$. (P.) hirtipes qui semble supporter des températures plus élevées.

b) Parmi les stations étudiées, citons deux exemples de pollution :

- dans la traversée du bourg de Laruns, on note une diminution de la richesse spécifique qui, de 7 espèces en amont (st. 27), passe à 3 en aval (st. 28), les espèces restantes (S. (Od.) ornatum, S. (S.) variegatum et $S$. (S.) argyreatum) étant réputées polluorésistantes.

- pour la même raison, la richesse spécifique du Valentin en amont de la station de sports d'hiver de Gourette (st. 18) passe de 7 espèces à 5 (en aval, st. 19).

Disparaissent $S$. (N.) cryophilum, P. (P.) hirtipes et pratiquement $S$. (N.) carthusiense. L'abondance des espèces restantes : S. (S.) variegaium, S. (S.) argyreatum et $S$. (S.) monticola diminue notablement. Rap. pelons ici le rôle important joué par les Simulies comme indicateurs biologiques dans la surveillance des cours d'eau de montagne.

\section{Ecologie des espèces dominantes du peuplement}

Les données faunistiques semi-quantitatives (Tableau II) ont été traitées dans deux analyses factorielles des correspondances (A.F.C.) et avec la méthode des nuées dynamiques afin de préciser la distribution spatiale et l'écologie des espèces.

\subsection{Les paramètres des stations}

D'après Angelier et al. (1985), cinq paramètres seulement suffisent pour rendre compte de la distribution spatiale des Hydracariens dans les cours d'eau de montagne : l'altitude de la station, l'altitude de la source la plus en amont sur le même cours d'eau, la pente, la surface du bassin-versant et le régime des eaux. Afin d'étudier la répartition des Ephémères en vallée d'Ossau, Vinçon \& Thomas (1987) ont ajouté à cet ensemble 5 autres paramètres précisant l'environnement de la station : alcalinité, conductivité, numéro d'ordre du cours d'eau, vitesse du courant et température maximale. Ces 10 descripteurs du milieu lotique montagnard ont également été choisis par nous-mêmes pour décrire les biotopes des stations.

Une première analyse a traité deux ensembles de données: les 29 stations et les 10 paramètres subdivisés en 47 classes (Tableau IV), une deuxième a traité deux autres ensembles : les 29 stations et les 27 taxons.

\subsection{A.F.C. stations - paramètres}

Les quatre premiers axes de la figure 2 rendent compte de $53 \%$ de l'inertie totale du nuage des points.

Pour faciliter l'interprétation de la structure des axes $F 1$ et $F 2$, les valeurs de cinq paramètres principaux sont reliées entre elles.

\section{SIGNIFICATION DES AXES}

La structure F1-F2 montre un effet Guttman assez net, révèlant un phénomène sériel; en effet, les points se disposent approximativement selon un gradiant constitué par un arc de parabole ; les extrémités du nuage (cornes) renferment les éléments les plus " spécialisés", à savoir :

$\rightarrow$ d'une part, les ruisseaux lents et chauds de piémont,

- d'autre part, les ruisseaux et torrents froids de haute altitude.

Dans la région centrale ou médiane de la parabole se rassemblent les éléments les plus moyens, relativement à l'échantillon.

* L'axe F1 paraît déterminé principalement par la vitesse du courant et à un moindre degré par les paramètres physico-chimiques. Des ruisseaux lents à forte conductivité s'opposent aux autres stations 
Tableau IV. Les parametres pris en compte dans la première analyse factorielle des correspondances. 47 classes.

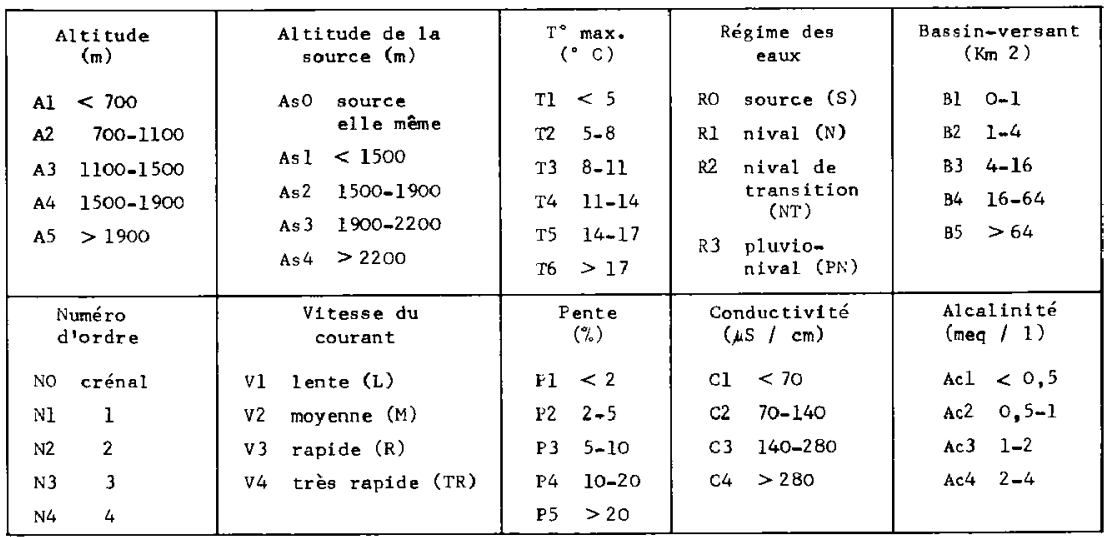

caractérisées par des vitesses rapides et des conductivités réduites.

* L'axe F2 reflète principalement le numéro d'ordre décroissant ; les cours d'eau de haute altitude ainsi que leurs stations s'opposent à ceux de la basse et moyenne vallée.

* L'axe F3 n'a pas été représenté ; il apporte pet1 de renseignements nouveaux par rapport aux deux premiers, sinon un isolement important du crénal.

Notons qu'aucun des axes 1 à 4 ne rend compte de façon satisfaisante de la température ; en revanche, les points $\mathrm{T} 1, \mathrm{~T} 2, \mathrm{~T} 3, \mathrm{~T} 4$ et $\mathrm{T} 5$, non reliés sur la figure, suivent approximativement l'arc de la parabole (avec toutefois une inversion dans la région médiane, entre T3 et T4), ce qui souligne le rôle important joué par ce paramètre.

\subsection{A.F.C. stations-espèces}

Les quatre premiers axes de la figure 3 rendent compte de $53 \%$ de l'inertie du nuage des points; la signification des axes F1 et F2 est assez semblable à celle donnée dans l'analyse précédente.

\section{SIGNIFICATION DES AXES}

* Axe F1. Sur cet axe, s'opposent nettement des espèces très rhéophiles $(P$. (P.) rufipes, $P$. (P.) latimucro, S. (N.) bertrandi, S. (N.) carthusiense...) et des espèces de biotopes plus lents ( $S$. (Od.) ornatum, S. (Od.) nitidifrons, S. (E.) gr. aureum, S. (N.) latigonium...), regroupées près des stations 23,24 et 25 .

* Axe F2. Certaines especes positives sur cet axe : $S$. (Od.) gr. ornatum (forme particulière rencontrée dans le Rio Gallego à $1750 \mathrm{~m}$ ), $P$. ( $P$.) tomosvaryi, $P$. (P.) latimucro, $S$. (N.) brevidens, apparaissent plus crénophiles que les autres; elles se développent le plus souvent dans des ruisseaux de petites dimensions ou aux abords des sources et s'opposent à des éléments qui colonisent de préférence les cours d'eau de plus grandes dimensions $S$. (S.) argenteostriatum, le groupe monticola, S. (S.) variegatum..., situés en position négative sur l'axe F2.

* Axe F3. (non représenté).

Comme dans l'analyse précédente, une forte individualisation des sources est observée sur cet axe (st. 14 et 21 ), par rapport aux ruisseaux de moyenne et haute montagne.

\section{GROUPEMENTS D'ESPECES ET DE STATIONS}

Trois groupements sont nettement visibles sur le plan des axes $\mathbf{F} 1$ et $\mathbf{F} 2$ de l'analyse stations-espèces. Nous distinguons les espèces « Fondamentales » d'un biotope qui n'appartiennent qu'à un groupement et 


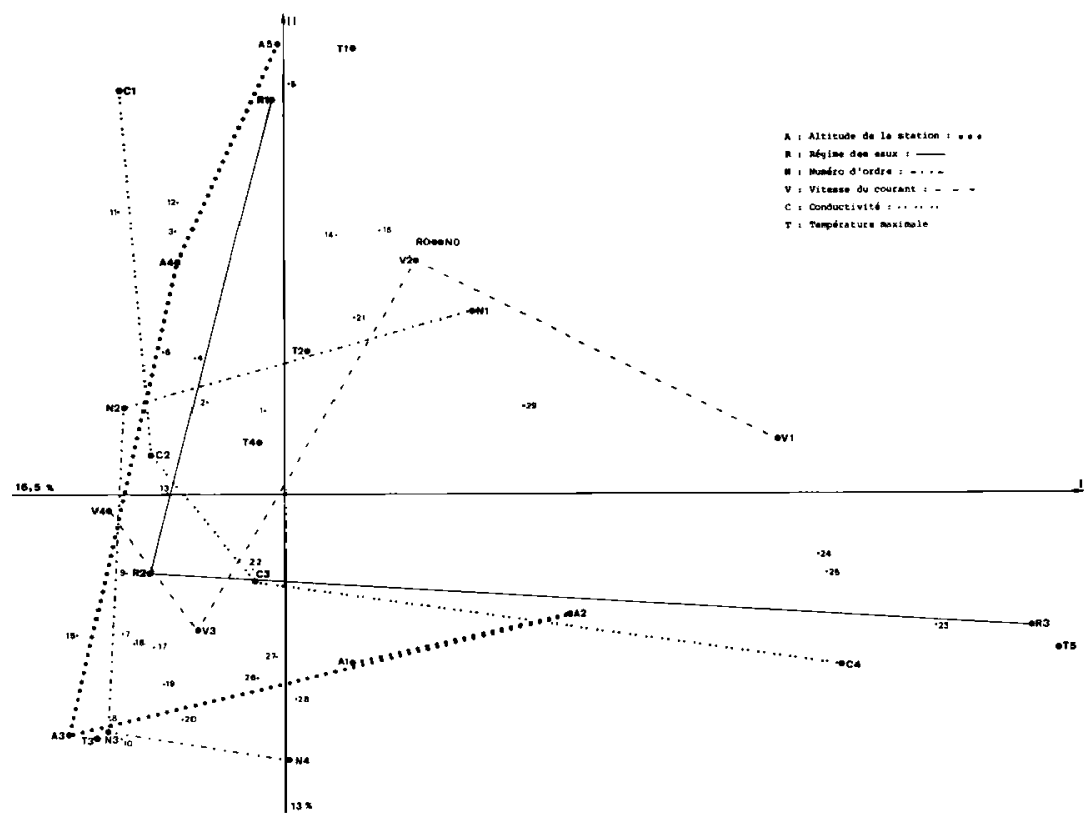

Fig. 2. Analyse factorielle des correspondances stations-paramètres. Evolution de quelques parametres importants sur les axes F1 et F2.

les espèces * accessoires *, plus eurytopes, qui peuvent appartenir aussi à d'autres groupements.

- GROUPEMENT I : sources et ruisseaux de moyenne et haute montagne

Espèces fondamentales

Espèces accessoires
P. (P.) tomosvaryi
$P$. (P.) hirtipes
$P$. (P.) latimucro
P. (P.) rufipes
$S$. (N.) brevidens
$S$. (N.) bertrandi
$S$. (N.) carthusiense forme $S$. (N.) carthusiense truncatum
S. (Od.) spinosum
S. (Od.) gr. ornalum
S. (S.) argyreatum
(forme de Rio Gallego, $1750 \mathrm{~m}$ )
Il est difficile de caractériser l'écologie des espèces fondamentales de ce groupement : elle sont peu fréquentes en vallée d'Ossau et à tendance sténotherme d'eau froide.

Stations représentatives: 14,21 et 1 .

Biotope : sources et ruisseaux de petites dimensions en moyenne ou haute mont agne ; vitesse du courant modérée à rapide, régime nival à nival de transition ; ces espèces supportent des températures estivales basses ( 5 à $7^{\circ} \mathrm{C}$ ), mais certaines peuvent aussi se développer dans des ruisseaux plus chauds en haute altitude (st. 1, 2, 3, 4 et 12).

-- GROUPEMENT II : torrents de montagne à courant rapide et fond rocheux. 


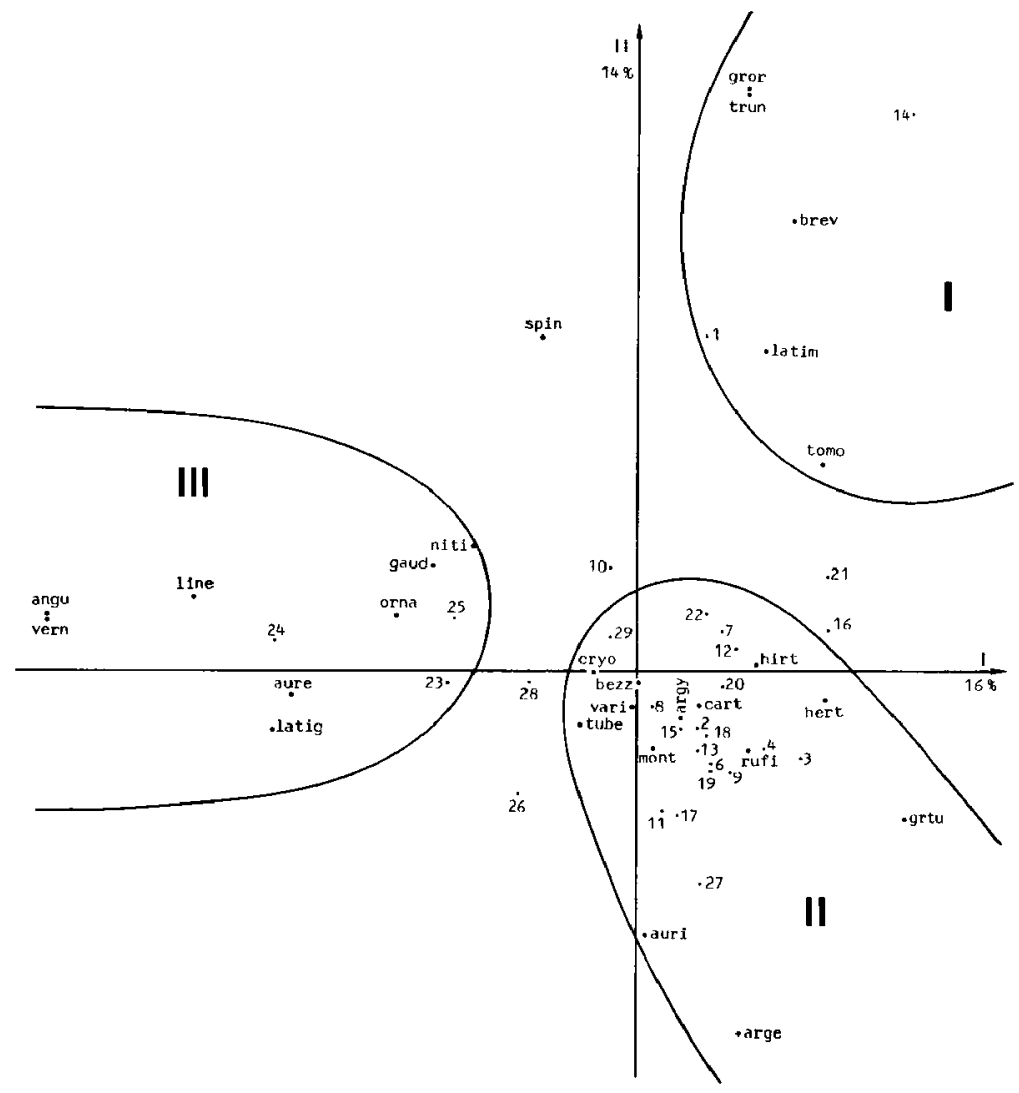

Fig. 3. Analyse factorielle des correspondances stations-espèces. Groupements d'espèces sur les axes F1 et F2.

Les stations sont indiquées par les chiffres arabes, les groupements par les chiffres romains et les espèces par les 4 ou 5 premières lettres de leur nom. 
Espèces fondamentales

Espèces accessoires

$P$. (P.) rufipes

P. (P.) latimucro

$P$. (P.) hirtipes

S. (S.) argenteostriatum

$S$. (N.) carthusiense

$S$. (N.) cryophilum

S. (Ob) auricoma

$S$. $(N$.) bertrandi

S. (S.) argyreatum

S. (S.) monticola

S. (S.) variegatum

S. (T.) bezzii

Les espèces fondamentales de ce groupement sont très rhéophiles et plus eurythermes que celles du groupe précédent ; la plupart d'entre elles sont fréquentes et abondantes dans tout le milieu lotique montagnard, depuis le piémont $(500 \mathrm{~m})$ jusqu'en altitude (1 $800 \mathrm{~m})$.

Stations représentatives : $2,3,4,6,8,9,11,12,13$, $15,17,18,19,20,22,27$.

Biotope : torrents de montagne ; courant rapide à violent, régime nival de transition, température maximale de 9 à $15^{\circ} \mathrm{C}$ (suivant l'altitude et l'origine des eaux).

- Groupement III : ruisseaux à faible courant, riches en macrophytes

\section{Espèces fondamentales}

Espèces accessoires

S. (E.) gr. aureum

S. (Od.) spinosum

$S$. (Od.) nitidifrons

P. (P.) hirtipes

S. (Od.) omatum

S. (N.) latigonium

S. (S.) argyreatum

S. (S.) monticola

S. (N.) angustitarse

S. (S.) variegatum

S. (W.) lineatum

S. (S.) gaudi

S. (N.) vermum.

Curieusement, $S$. (N.) vermum (en synonymie avec l'ancienne dénomination $S$. latipes) se retrouve ici dans les milieux lents alors que l'espèce vit souvent dans la partie amont de ruisseaux peu profonds à courant assez vif ou modéré. Cependant, Dorier (1962-63) l'avait également récoltée dans de larges ruisseaux à courant modéré, riches en végétation phanérogamique.

Certaines espèces fréquentes et abondantes dans les faciès rapides $P$. ( $P$.) hirtipes, S. (S.) argyreatum...) se développent aussi, mais en bien moindre abondance, dans les faciès lents ; c'est pourquoi nous les avons considérées comme accessoires. $S$. (S.) gaudi et $S$. (Od.) spinosum caractérisent plus particulièrement les biotopes calcaires.
Stations représentatives : 23, 24, 25 .

Biotope : ruisseaux à cou rant lent ou modéré, riches en macrophytes; zone de piémont (altitude inférieure à $900 \mathrm{~m}$ ), températures estivales élevées (de 15 à $20^{\circ} \mathrm{C}$ ); régime pluvio-nival et fortes teneurs en calcium.

\section{Discussion et conclusion}

A cause de sa forte rhéophilie, la communauté simulidienne de la vallée d'Ossau possède une structu re relativement simple, surtout si elle est comparèe à celle d'autres groupes d'Invertébrés de cette même vallée, tels que les Ephémères (Vinçon \& Thomas 1987).

Trois groupements apparaissent nettement isolés :

Le premier (I) caractérise les sources et les ruisseaux de moyenne et haute montagne ; il est très peu diversifié à cause de la rareté des espèces strictement sténothermes d'eau froide.

Une grande opposition existe entre les groupements II et III qui correspondent à deux biotopes fort différents, II : torrents rapides à fond rocheux, régime nival de transition et températures estivales relativement basses (larves et nymphes sur les rochers) et III : ruisseaux lents à fond rocheux partiellement envasé, régime pluvio-nival et températures estivales élevées (larves et nymphes sur la végétation ou les rochers). Comme l'a montré la première analyse factorielle (stations-paramètres), c'est surtout la vitesse du courant qui oppose ces deux derniers biotopes; ce facteur détermine d'ailleurs partiellement la nature du substrat et la température de l'eau, toutes deux fonction de la vitesse d'écoulement.

Le régime des eaux, nival de transition ou pluvionival, est sans doute aussi responsable en partie de cette opposition, comme cela a été déjà observé en vallée d'Ossau (Ephémères: Vinçon \& Thomas op. cit.) eł en vallée d'Aure (Hydracariens: Angelier et al. 1985). Ce facteur influe entre autre sur le regime thermique du cours d'eau et sur la stabilité du substrat (crues de fonte des neiges plus ou moins violentes en régime nival de transition).

Cet te structure est confirmée d'une part grâce à la méthode des nuées dynamiques (Tableau V) et d'autre part, à l'aide d'une classification hiérarchique des espèces (fig. 4), réalisée sur les 3 premiers 
Tableau V. Méthode des nuées dynamiques. (3 axes). Répartition des espèces et des stations en quatre classes.

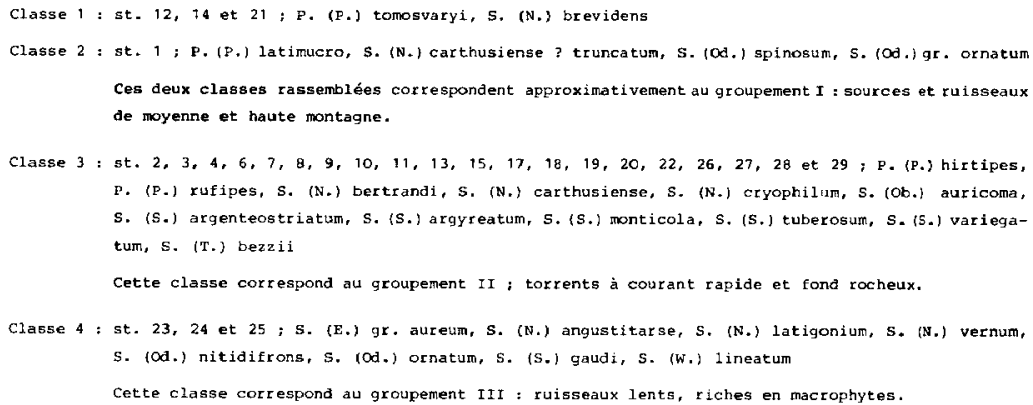

facteurs de l'A.F.C. stations-espèces ; (distance euclidienne et moment d'ordre 2).

Quatre ensembles correspondant aux quatre classes des nuées dynamiques s'individualisent nettement dans cette hiérarchisation. Nettement différenciés, les deux premiers ensembles (comme les deux premières classes des nuées dynamiques) sont toutefois assez imbriqués sur le plan des axes F1-F2 de l'A.F.C. stations-espèces; de ce fait, ils correspondent au seul groupement I des sources et ruisseaux de moyenne et haute montagne (fig. 3).

Les deux autres ensembles correspondent aux groupements II et III de l'A.F.C. et se révèlent par conséquent particulièrement stables. Les caractéristiques écologiques des populations de Simulies des groupements II et III sont déjà précisées dans des travaux antérieurs ; ainsi, Dorier (1962-63) oppose notamment les " cours d'eau herbeux à courant relativement faible et régulier (groupement III) et les cours d'eau à courant plus vif et fond pierreux dépourvus de végétation phanérogamique "(II).

D'un point de vue faunistique, un complément essentiel est apporté à la connaissance des Simulies de l'Ouest-pyrénéen, connaissance limitée jusqu'à présent à certains ruisseaux de basse altitude.

Dans son ensemble, le peuplement est assez semblable à celui des autres régions pyrénéennes, d'où une certaine homogénéité dans la répartition altitudinale. Cependant, nous retiendrons quelques caractéristiques propres à la région

1) la remontée en altitude d'espèces eurythermes $S$. (S.) variegatum et $S$. (T) bezzii, qui atteignent ici des altitudes nettement supérieures à celles des Pyrénées centrales, à cause d'une influence maritime accentuée ou de l'orientation Sud des cours d'eau :

2) l'absence de quelques éléments vivant en piémont dans les Pyrénées centrales et qui se dévelop. pent sans doute à des altitudes plus basses. $S$. ( $S$.) reptans a d'ailleurs été retrouvée récemment dans le Gave d'Oloron, situé en aval du Gave d'Ossau (Oloron-Sainte-Marie, $200 \mathrm{~m}$ ).

3) la présence d'espèces rares, ou absentes dans les autres régions pyrénéennes $S$. (N.) latigonium, $S$. (S.) gaudi, S. (S.) hispaniola...).

A l'avenir, il serait intéressant de prospecter de façon approfondie un réseau des PyrénéesOrientales avec des sources en haute altitude. Ceci, en complément du travail déjà réalisé sur la rivière Massane (altitude de la source $1000 \mathrm{~m}$ ) où les espèces sont fortement inféodées au clinat méditerranéen, notamment en zone de piémont.

Une telle prospection confirmerait sans doute une fois de plus la remontée en altitude d'espèces eurythermes, à mesure que le climat s'adoucit et que la température devient moins rigoureuse, avec un régime des eaux à dominance pluvio-nival. 


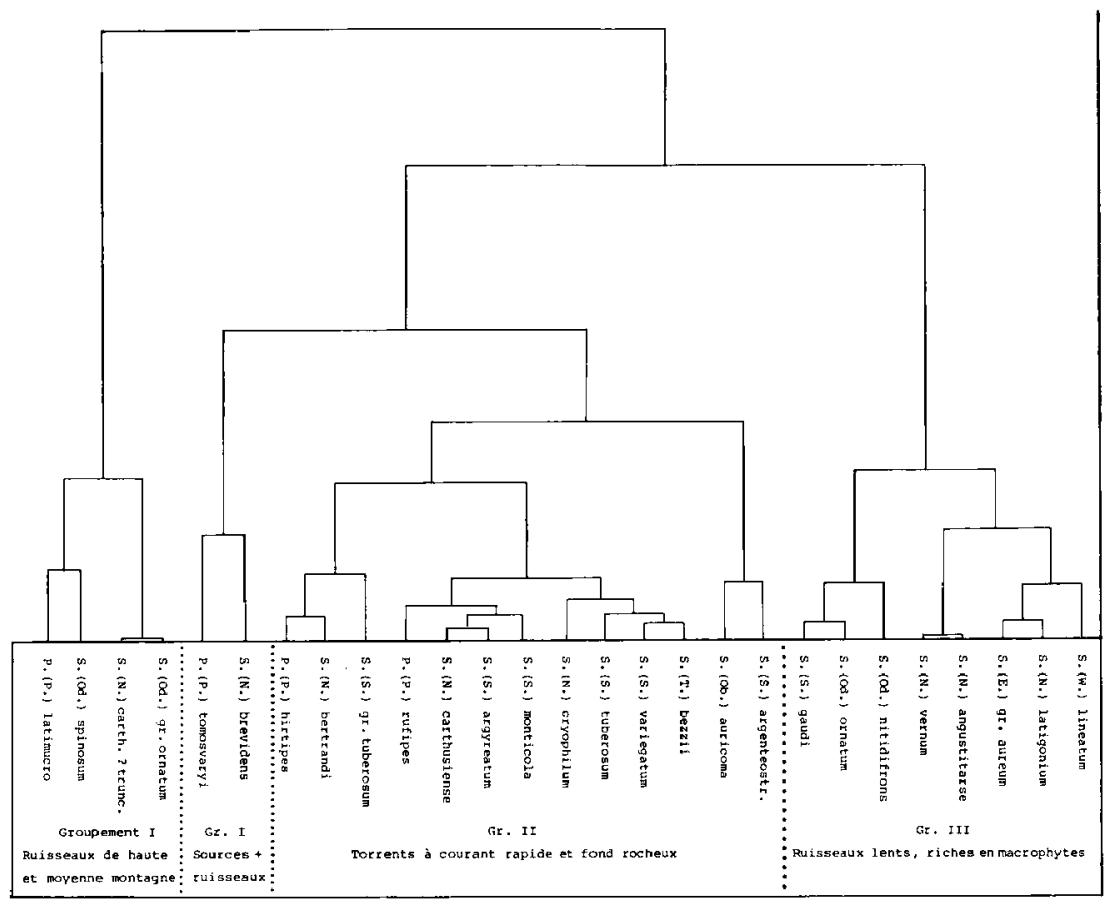

Fig. 4. Classification hiérarchique des especes sur les 3 premiers axes de l'analyse factorielle des correspondances.

\section{Remerciements}

Nous remercions cordialement Monsieur L. Bonnet (Biologie quantitative, Toulouse) pour sa précieuse contribution à ce travail lors du traitement des données et Made. moiselle F. Baurès pour son aide technique.

\section{Travaux cités}

Angelier (E.), Angelier (M.L.) \& Lauga (J.) 1985. - Recherches sur l'écologie des Hydracariens (Hydrachnellae, Acarï) dans les caux courantes. Annls Limnol, 21 (1): 25-64.

Beaucournu-Saguez (F.). 1975. - Sur quelques Simulies (Diptera, Simuliidae) du Nord-Ouest de l'Espagne. Ann. Parasit., 50 (1) 105-122.
Clergue-Gazeau (M.). 1987. - Influence du climat méditerranéen sur la faune sirnulidienne (Diptera, Nematocera) d'une rivière pyrenénne : la Massane. Ecologia Mediterramea, 13 (1-2) : 3-13.

Clergue-Gazeau (M.) \& Gazagnes (G.). 1986. - Les Simuliidae de la Neste d'Aure (Pyrénées centrales). I. Impact des aménagements hydrólectriques dans la haute vallée. (Diptera, Nematocera). Annls Limnol., 22 (2): 195.203.

Clergue-Gazeau (M.), Holmière (D.), Meurgues (S.) et Angelier (E.). 1987. - Les Simuliidae (Diptera, Nematocera) de la Neste d'Aure (Pyrénées centrales). II. Notes écologiques et impact des aménagernents dans la basse vallèe. Annls Limnol, 23 (3) : 197-207.

Crosskey (R.W.1981. - Simulid Taxonomy. The contemporary scene. Blackflies : 3-18. In : The future of biological methods in integrared cont rol. Laid M., ed. Academic press. London. 399 p.

Doby (J.M.), David (F.) \& Rault (B.). 1964. - Nouvelles stations françaises (Morbihan et Dordogne) de Simulium rupicolum forme hispaniola Grenier \& Bertrand, 1954. Comparaison de la répartition géographique avec celle de l'espece type Séguy \& Dorier, 1936 (1). Ann. Parasit., 39 (4) : 515-526 
Doby (J.M.) \& Deblock (S.). 1955. - A propos de Simulium (Prosi. mulium) hirtipes Fries 1824 : observations morphologiques et biologiques. Stations nouvel les pour la variété arvernense $\mathrm{G}$ re. nier 1947. Ann. Parasit., 30 (3): 272-277.

Dorier (A.). 1962-1963. - Documents pour servir à la connaissance des Simulidae du Sud-Est de la France. Trav. Lab. Hydrob. Pisc. Grenoble., $54.55: 7.79$.

Gagneur (J.). 1976. - Répartition des Simuliidae (Diptera) dans le Lot. Annis Limnol., 12 (3) : 287-298.

Gazagnes (G.). 1983. - Contribution a t'étude de la derive des Invertébrés sur la haute Neste d'Aure; impact des aménage ments hydro-étectriques. Thèse de Doctorat de 3eme cycle. Toulouse. 104 p. + annexe.

González (G.). 1980. - Primeres dades sobre la distribucio dels Simuliidae (Diptera, Nematocera) d'Andorra. Bull. Inst. Cat. Hist. Nat., 45 (Sec. zool., 3) : 97-106.

González-Pena (G.). 1983. -- Factores que determinan la distribu cion de los simulidos y quironomidos (Diptera) de la red hidrografica andorrana. Actas del Primer Congreso Espagnol de Lim. nologia. 1: 197-205.

Jarry (D.M.). 1973. - Contribution à l'étude des Simulies du Languedoc-Roussillon II. De quelques espèces des Pyrénèes Orientales. Ann. Parasitol. Humaine et Comp. 48 (5) : 703-712

Jarry (D.M.). 1975. - Contribution à l'étude des Simulies du . Languedoc-Roussillon III. A propos de six espèces nouvelles pour les Pyrénées-Orientales. Vie et Milieu., 25 (2) : 249.258.

Lavandier (P.). 1976. - Premières données sur la répartition des Simuliidae (Diptères. Nématocères) dans la vallèe d'Aure (Hautes-Pyrénées, France). Bull. Soc. Hist. Nat. Toulouse, 112 (1.2): 89.95
Lavandier (P.). 1979. - Ecologie d'un torrent pyrénéen de haute montagne: l'Estaragne. These de Doctorat d'Etat, Toulouse. $532 \mathrm{p}$.

Neveu (A.). 1970. - Ecologie des Simulitade (Diptera, Nermatocera) d'un ruisseau des Byrénées-Atlantiques: le Lissuraga. These Doctorat 3ème cycle. Fac. Sci. Univ. Paris, 109 p.

Neveu (A.) \& Lapchin (L.). 1979. - Ecologie des principaux invertébrés filtreurs de la basse Nivelle (Pyrénées-At lantiques). I. Simulidae (Diptera, Nematocera). Annls. Limnol., 14 (3) : 225-244.

Vinçon (G.). 1987a. - Comparaison de la faune benthique des vallées d'Aure et d'Ossau, en vue de l'elaboration d'une théthodo. logie de surveillance des cours d'eau de montagne. Thèse de Docteur-Ingénieur. Toulouse, $n^{\circ} 960.381 \mathrm{p}$.

Vinçon (G.). 1987b. - Etude hydrobiologique de la vallée d'Ossau (Pyrénées-Atlantiques) II. Le milieu et la structure du peuplement. Annls. Limnol., 23 (3): 225-243.

Vinçon (G.) \& Thomas (A.G.B.). 1987. - Etude hydrobiologique de la vallée d'Ossau (Pyrénées-Atlantiques). I. Répartition et éco logie des Ephéméroptères. Annls Limnol., 23 (2) : 95-113.

Zwick (H.). 1974. - Faunistisch-ökologische und taxonomische Untersuchungen an Simuliidae (Diptera), unter besonderer Berucksichtigung der Arten des FuldaGebietes. Abhand. Senck. Natur. Ges., 533: 1-116.

Zwick (H.). 1978. - Simuliidae. In Ill ies (J.) ed. : Limnofauna Euro paea. Fischer Verlag. Stuttgart : $396-403$ 